\title{
Ventile auch bei sehr schwerem homogenem Emphysem wirksam
}

Fragestellung: Profitieren Patienten trotz sehr niedriger $\mathrm{FEV}_{1}$ von einer endoskopischen Lungenvolumenreduktion (ELVR)?

Hintergrund: Patienten mit $\mathrm{FEV}_{1}<20 \%$ und homogenem Emphysem oder niedriger Diffusionskapazität (DLCO) weisen eine erhöhte Mortalität nach chirurgischer Lungenvolumenreduktion (LVRS) auf [1]. Als alternative minimalinvasive Technik stellt die ELVR mittels Ventilimplantation eine Therapieoption dar, die ebenso wie die LVRS das Ziel der Reduktion der Hyperinflation verfolgt, jedoch eine

\section{Originalie}

Trudzinski FC, Höink AJ, Leppert $D$ et al. Endoscopic Lung Volume Reduction Using Endobronchial Valves in Patients with Severe Emphysema and Very Low FEV1. Respiration. 2016;92(4):258-65. geringere Morbidität und Mortalität aufweist [2].

Patienten und Methoden: In die retrospektive Analyse wurden 20 konsekutive Patienten mit $\mathrm{FEV}_{1} \leq 20 \%$ und niedriger DLCO aufgenommen, die von Juni 2012 bis Januar 2015 mit endobronchi- alen Ventilen behandelt wurden. Ergebnisse der Lungenfunktionsparameter und des 6-Minuten-Gehtests (6-MWT) vor und vier bis sechs Wochen nach dem Eingriff wurden verglichen und die Rate unerwünschter Ereignisse erhoben.

Ergebnisse: Die Lungenfunktion verbesserte sich nach der Ventilimplantation signifikant: die $\mathrm{FEV}_{1}$ stieg von 16,97 auf 21,03\%. Das Residualvolumen (RV) sank von 322 auf $270 \%$. Auch im 6-MWT ließ sich eine im Mittel gestiegene Belastbarkeit der Patienten erkennen (von 239 \pm 77 auf $267 \pm 97$ Meter). Ein Pneumothorax, der die häufigste Komplikation der Ventiltherapie darstellt, trat bei 5 der 20 Patienten (25\%) auf. Die 30-Tage-Mortalität betrug $0 \%$.

Schlussfolgerung: Die ELVR stellt auch bei Hochrisikopatienten mit sehr geringer $\mathrm{FEV}_{1}$ ein gut durchführbares und relativ sicheres Verfahren dar. Der Pneumothorax ist mit einer Rate von $25 \%$ die häufigste Komplikation. Die Therapie führt zu einer moderaten, jedoch signifikanten Verbesserung der Lungenfunktionsparameter und zu einer Zunahme der Belastbarkeit.

\section{- Kommentar von Dr. Konstantina Kontogianni \\ Immer individuell abwägen!}

Die Studie von Trudzinski et al. ist eine retrospektive Analyse von 20 sorgfältig ausgewählten Patienten mit schwerstgradi-

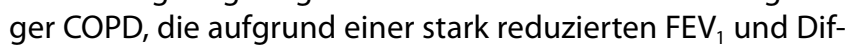
fusionskapazität nach Kriterien der NETT-Studie zu Hochrisikopatienten für eine LVRS zählen. Die Analyse zeigte, dass eine endoskopische Ventiltherapie auch bei dieser Kohorte die lungenfunktionellen Parameter und die Belastbarkeit verbessern kann. Als häufigste Komplikation trat ein Pneumothorax mit einer Rate von $25 \%$ auf. Dennoch ist die Ventiltherapie mit einer hier erhobenen Mortalität von $0 \%$ im Vergleich zur Chirurgie, bei der die 30-Tages-Mortalität $16 \%$ betrug [3], als sicheres Verfahren anzusehen.

Die Lungenfunktion der Patienten verbesserte sich nach

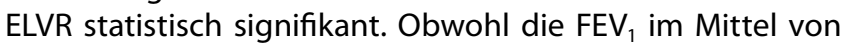
$110 \mathrm{ml} \pm 150 \mathrm{ml}$ anstieg, erreichten nur $25 \%$ der Patienten ein klinisch relevante Verbesserung von $>100 \mathrm{ml}$. Das Residualvolumen (RV) nahm im Mittel um $1.100 \mathrm{ml} \pm 1.640 \mathrm{ml}$ ab. Hier ließ sich bei $58 \%$ der Patienten eine klinische Verbesserung mit einer RV-Abnahme von $430 \mathrm{ml}$ feststellen. Dabei profitierten $\mathrm{Pa}$ tienten mit heterogener Emphysemverteilung oder einer lobären Atelektase nach Ventilimplantation am meisten.

Als Nachteil der Studie ist die geringe Patientenzahl zu erwähnen. Es sind weitere, auch prospektive Studien nötig, um die Effektivität der Ventiltherapie in dieser speziellen Patientenkohorte zu evaluieren. Aufgrund des kurzen Beobach-

tungsintervalls von vier bis sechs Wochen nach ELVR können keine Aussagen über den Langzeitverlauf gemacht werden.

Einschränkend muss darauf verwiesen werden, dass es bislang keine Daten gibt, die eine ELVR mit der LVRS direkt vergleichen, sodass dazu keine definitive Aussage möglich ist. Patienten mit schwerer COPD und Überblähung sollten daher interdisziplinär diskutiert werden, um für den Einzelnen das beste Verfahren zur Lungenvolumenreduktion zu finden.

\section{Literatur:}

1. Fishman A, Martinez F, Naunheim K et al. N Engl J Med. 2003;348:2059-73

2. Sciurba FC, Ernst A, Herth FJ et al. N Engl J Med. 2010;363:1233-44

3. Kaplan RM, Sun Q, Naunheim KS, Ries AL. Ann Thorac Surg. 2014;98:1782-9

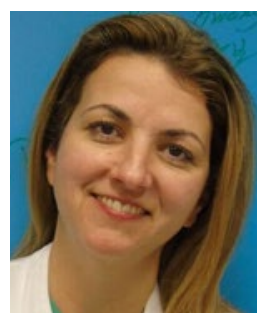

Dr. Konstantina Kontogianni

Pneumologie und Beatmungsmedizin, Thoraxklinik, Universitätsklinikum Heidelberg, Deutschland. Röntgenstraße 1, 69126 Heidelberg Konstantina.Kontogianni@med. uni-heidelberg.de 